\title{
Desarrollo comparativo de dos especies Inga en base a su potencial agroforestal
}

Comparative development of two Inga species based on their agroforestry potential

Desenvolvimento comparativo de duas espécies de Inga com base em seu potencial agroflorestal

- Allan Alberto Alvarado Aguayo

aalvarado@uagraria.edu.ec

Código ORCID: 0000-0003-2245-7661

Universidad Agraria del Ecuador, Ecuador

- Wilmer Omar Pilaloa David

wpilaloa@uagraria.edu.ec

Código ORCID: 0000-0002-0653-552X

Universidad Agraria del Ecuador, Ecuador
- Braulio Javier Carrera Maridueña

bcarrera@uagraria.edu.ec

Código ORCID: 0000-0002-5003-743X

Universidad Agraria del Ecuador, Ecuador

- Dolores Mariela Carrera Maridueña

dcarrera@uagraria.edu.ec

Código ORCID: 0000-0002-9101-7435

Universidad Agraria del Ecuador, Ecuador

Artículo recibido 31 de agosto 2020 / Arbitrado y aceptado 28 de octubre 2020 / publicado 18 de diciembre 2020

\section{RESUMEN}

Las especies de guabo Inga edulis e I. vera son ampliamente utilizadas en sistemas agroforestales. Este estudio compara sus indicadores de desarrollo fenológico, aporte de minerales y presencia de organismos benéficos en su entorno de influencia, mediante un diseño de bloques completamente al azar (DBCA) con tres tratamientos y seis repeticiones. I. edulis tiene crecimiento polinomial más promisorio, con $5.79 \mathrm{~m}$ de altura, diámetro altura de pecho (DAP) de $0.075 \mathrm{~m}$ y 31.66 ramas con un diámetro de sombra de $5.94 \mathrm{~m}$, superando a I. vera que alcanza $4.74 \mathrm{~m}$ de altura, DAP de $0.061 \mathrm{~m}$ y 14.33 ramas con sombra diametral de $4.79 \mathrm{~m}$. Ambas especies difieren en su aporte de macronutrientes al suelo, superando I. vera en $\mathrm{NH} 4, \mathrm{~K}$ y $\mathrm{Mg}$, mientras que $\mathrm{I}$. edulis realiza un mayor aporte de $\mathrm{P}$ y Ca. El contenido mineral foliar también difiere, superando I. edulis en $\mathrm{NH} 4, \mathrm{P}$ y $\mathrm{Mg}$ foliares, no obstante, I. vera contiene mayor $\mathrm{K}$ y Ca foliares. En la hojarasca, I. edulis posee $3.13 \% \mathrm{~N}$, mientras que I. vera 2,53\% N. La fauna asociada a estas especies se relaciona con la presencia de lombrices de tierra (Lumbricus terrestres) en el área del subsuelo con influencia de la raíz, existiendo mayor promedio de individuos por árbol en I. edulis (22.6) que en I. vera (14.9). Sin embargo, en I. vera existe mayor población de avispas Polistes dominula (4.6) y Vespula vulgaris (10.3), con relación a I. edulis, que alcanza 2.7 y 5.2 individuos por árbol, respectivamente.

Palabras clave: Biomasa forestal; fabácea; fauna benéfica; desarrollo fenológico; leguminosa; sistema agroforestal

\begin{abstract}
The species of Inga edulis and I. vera are widely used in agroforestry systems. This study compares their phenological development indicators, mineral contribution and presence of beneficial organisms in their influence environment, through a completely randomized block design (CRBD) with three treatments and six repetitions. I. edulis has more promising polynomial growth, with $5.79 \mathrm{~m}$ height, diameter breast height (DBH) of $0.075 \mathrm{~m}$ and 31.66 branches with a diameter of $5.94 \mathrm{~m}$ shadow, surpassing I. vera that reaches $4.74 \mathrm{~m}$ height, DBH of $0.061 \mathrm{~m}$ and 14.33 branches with diametric shadow of $4.79 \mathrm{~m}$. Both species differ in their contribution of macronutrients to the soil, exceeding I. vera in $\mathrm{NH} 4, \mathrm{~K}$ and $\mathrm{Mg}$, while I. edulis makes a greater contribution of $\mathrm{P}$ and Ca. Foliar mineral content also differs, with I. edulis exceeding $\mathrm{NH} 4, \mathrm{P}$ and $\mathrm{Mg}$ in foliar, but I. vera contains more $\mathrm{K}$ and $\mathrm{Ca}$ in foliar. In the leaf litter, I. edulis has $3.13 \% \mathrm{~N}$, while I. vera has $2.53 \% \mathrm{~N}$. The fauna associated with these species is related to the presence of earthworms (Lumbricus terrestris) in the subsoil area with root influence. There is a higher average number of individuals per tree in I. edulis (22.6) than in I. vera (14.9). However, in I. vera there is a larger population of Polistes dominula (4.6) and Vespula vulgaris (10.3), in relation to I. edulis, which reaches 2.7 and 5.2 individuals per tree, respectively.
\end{abstract}

Key words: Forest biomass; fabaceae; beneficial fauna; phenological development; leguminous; agroforestry system 


\section{RESUMO}

As espécies de Inga edulis e I. vera são amplamente utilizadas em sistemas agroflorestais. Este estudo compara seus indicadores de desenvolvimento fenológico, fornecimento mineral e presença de organismos benéficos em seu ambiente de influência, utilizando um desenho de blocos completamente aleatórios (DBCA) com três tratamentos e seis réplicas. I. edulis tem o crescimento polinomial mais promissor, com uma altura de $5,79 \mathrm{~m}$, um diâmetro à altura do peito (DBH) de 0,075 m e 31,66 ramos com um diâmetro de 5,94 m, superando I. vera que atinge $4,74 \mathrm{~m}$ de altura, DBH de 0,061 $\mathrm{m}$ e 14,33 ramos com um diâmetro de 4,79 $\mathrm{m}$. As duas espécies diferem em sua contribuição de macronutrientes para o solo, excedendo I. vera em $\mathrm{NH} 4, \mathrm{~K} \mathrm{e} \mathrm{Mg}$, enquanto I. edulis faz uma contribuição maior de $\mathrm{P}$ e Ca. $\mathrm{O}$ conteúdo de minerais foliares também difere, com I. edulis excedendo NH4, P e Mg em foliar, entretanto, I. vera contém mais $\mathrm{K}$ e Ca. Na cama foliar, I. edulis tem $3,13 \% \mathrm{~N}$, enquanto I. vera tem $2,53 \% \mathrm{~N}$. A fauna associada a estas espécies está relacionada à presença de minhocas (Lumbricus terrestris) na área do subsolo com influência radicular, com um número médio maior de indivíduos por árvore em I. edulis $(22,6)$ do que em I. vera $(14,9)$. Entretanto, em I. vera há uma população maior de Polistes dominula $(4,6)$ e Vespula vulgaris $(10,3)$, em relação a I. edulis, que atinge 2,7 e 5,2 indivíduos por árvore, respectivamente.

Palavras-chave: Biomassa florestal; fabacea; fauna benéfica; desenvolvimento fenológico; leguminosas; sistema agroflorestal

\section{INTRODUCCIÓN}

La introducción de leguminosas arbustivas y arbóreas al agro ecosistema contribuye a mejorar las propiedades biológicas, químicas y físicas del suelo agrícola (1). Ello fomenta el desarrollo de la agricultura sostenible radica, pues con la asociación de cultivos y leguminosas las prácticas agrícolas convencionales se potencian dentro de un sistema de producción más viable en la parte ambiental, social y económica (2).

Todas las leguminosas en el hábitat edáfico contribuyen con la fijación biológica de nitrógeno atmosférico (3). Particularmente, en la agroforestería, los árboles fijadores de nitrógeno pueden asociarse con cultivos agrícolas, con pasturas, o también en sistemas agrosilvopastoriles, bancos forrajeros y cercas vivas (4), constituyéndose como árboles multipropósito que desempeñan un papel básico con múltiples beneficios al ecosistema, tanto de su entorno como del subsuelo (5). Estos árboles fijan el nitrógeno atmosférico en sus nódulos radicales $\mathrm{y}$, a través del metabolismo, lo almacenan en su componente forrajero: hojas, peciolos, tallos tiernos y frutos (6). Por medio del aporte de materia orgánica hecho al suelo a través de la caída periódica o estacional, natural o provocada (cosecha), de hojas, flores, frutos, ramas y raíces muertas, es incrementado el nitrógeno en el suelo, en el área de influencia de los árboles (7).

En el ámbito de la agricultura moderna, caracterizada por la problemática de pérdida de suelos debido al mal uso de la tierra que induce la erosión, degradación de los suelos, pérdida de nutrientes, y lleva a la desaparición de los bosques tropicales, la implementación de sistemas agroforestales representa una alternativa eficiente a las prácticas inadecuadas de alto impacto ambiental (8).

La utilización de las especies de Inga destaca para el establecimiento de los sistemas agroforestales en Latinoamérica tropical (9). Países Andinos, tales como Colombia, Ecuador y Perú presentan la mayor diversidad de especies dentro de este género (10), siendo muy valorado por su amplio uso como árbol de sombra de cultivos perennes, con beneficios relacionados con la aportación de nitrógeno, materia orgánica, preservación de la humedad y prevención de la erosión (11).

Dentro de los diversos géneros de árboles utilizados en la agroforestería, Inga es el más prominente en América Tropical, destacándose por ser capaz de sobrevivir y florecer en suelos pobres ácidos, tan típicos de la zona (12). Su uso 
es conveniente, ya que además de ser tolerante a suelos empobrecidos, posee un crecimiento rápido, con gran adaptabilidad ecológica $\mathrm{y}$ aporta elevadas cantidades de biomasa (13).

La experiencia ha demostrado que el manejo agroforestal fomenta el equilibrio ecológico, regulando el agro ecosistema. En base a ello, el presente trabajo compara el potencial agroforestal de dos especies del género Inga. La hipótesis se basa en que la influencia de las especies $I$. edulis e $I$. vera tiene efectos positivos sobre la cantidad de elementos aportados al suelo y sobre el establecimiento de organismos benéficos en el área de influencia de los árboles.

\section{MATERIALES Y MÉTODOS}

\section{Área de estudio}

Se evaluó el crecimiento fenológico, el aporte nutricional y la presencia de fauna benéfica en Inga edulis (guaba de bejuco) e I. vera (guaba de machete), analizando su potencial agroforestal en la Granja Experimental El Vainillo, del cantón El Triunfo, Guayas, Ecuador, asentada a 10 metros sobre el nivel del mar (msnm), en las coordenadas geográficas $2^{\circ} 20^{\prime} 35.8 " \mathrm{~S}$ y $79^{\circ} 31^{\prime} 58.3^{\prime \prime} \mathrm{W}$, con un suelo de textura arcillosa (Alfisol), clima monzón tropical, caracterizado por una estación lluviosa entre diciembre y mayo, y una estación seca entre junio y noviembre, y un rango de temperaturas medias que oscilan entre $20^{\circ} \mathrm{C}(\mathrm{el}$ mínimo en agosto) y $31{ }^{\circ} \mathrm{C}$ (el máximo en marzo-abril).

\section{Distribución de los tratamientos}

Los árboles de Inga fueron sembrados a un distanciamiento de $6 \mathrm{~m} \times 6 \mathrm{~m}$, siguiendo el diseño de bloques completamente al azar (DBCA) con tres tratamientos y seis repeticiones (Tabla 1 ).

Tabla 1. Tratamientos para analizar el desarrollo comparativo de Inga edulis e I. vera, en la zona El Vainillo, El Triunfo, Guayas, Ecuador

\begin{tabular}{cccc}
\hline Tratamiento & Descripción & $\begin{array}{c}\text { Total de plantas } \\
\text { guabo / parcela }\end{array}$ & $\begin{array}{c}\text { Total de guabos / } \\
\text { tratamiento }\end{array}$ \\
\hline $\mathrm{T} 1$ & Inga edulis (Guaba de bejuco) & 15 & 90 \\
$\mathrm{~T} 2$ & Inga vera (Guaba de machete) & 15 & 90 \\
\hline
\end{tabular}

\section{Evaluación de las variables}

Las variables, evaluadas bimensualmente en el segundo año de establecimiento, se relacionaron con factores en el desarrollo de los árboles: altura de planta $(\mathrm{cm})$, diámetro del tallo a la altura del pecho (cm), cantidad de ramaje, diámetro de área con sombreamiento $(\mathrm{cm})$. Además, se determinó la riqueza de nitrógeno en las hojas, el aporte edáfico de minerales, con énfasis en macronutrientes, en el área de influencia de los árboles a través de análisis del suelo. En todos los casos el criterio se aplicó a diez (10) plantas muestreadas de cada tratamiento con sus repeticiones en un periodo de dos (2) años y tomando los datos de las variables transcurrido el primer año de establecimiento. 
Para determinar el potencial agroforestal comparativo entre las especies de Inga, se consideraron los aspectos del desarrollo fenológico de los árboles como hospedero de organismos benéficos (lombrices de tierra, avispas), identificándolos mediante muestreo de campo, con observación sin captura, en el entorno de influencia de los árboles

\section{RESULTADOS Y DISCUSIÓN}

\section{Comportamiento fenológico comparativo entre Inga edulis e I. vera, en la zona El Vainillo, El Triunfo, Guayas, Ecuador}

Existen diferencias significativas para la altura de planta (m), entre las dos especies. $I$. edulis presenta una altura promedio de 5.79 $\mathrm{m}$ en el último muestreo, transcurridos dos años de establecimiento, superando a I. vera que alcanza $4.74 \mathrm{~m}$ (Tabla 2). El crecimiento observado en el ensayo corresponde a los parámetros usuales para el género Inga en este periodo de tiempo, considerando que $I$. edulis, alcanza alturas aproximadas entre de 4 a $5 \mathrm{~m}$ de alto y 3 a $4 \mathrm{~m}$ de copa con una edad fenológica de tres a cuatro años (14).

Tabla 2. Altura comparativa mensual de plantas (m), en el segundo año de establecimiento en dos especies de Inga.

\begin{tabular}{lccc}
\hline \multirow{2}{*}{ Fechas } & \multirow{2}{*}{ Muestreos } & \multicolumn{2}{c}{ Medias observadas } \\
& & T1 (I edulis) & T2 (I vera) \\
\hline $15 / 07 / 19$ & 1 & 3.82 & 3.13 \\
$15 / 08 / 19$ & 2 & 4.20 & 3.45 \\
$15 / 09 / 19$ & 3 & 5.08 & 4.17 \\
$15 / 10 / 19$ & 4 & 5.21 & 4.28 \\
$15 / 11 / 19$ & 5 & 5.59 & 4.59 \\
$15 / 12 / 19$ & 6 & 5.79 & 4.75 \\
\hline
\end{tabular}

La especie Inga edulis tiene una línea de tendencia de crecimiento polinómica con un $\mathrm{R}^{2}$ $=0.9741$, a su vez la ecuación que expresa su crecimiento es $\mathrm{y}=-0.0518 \mathrm{x}^{2}+0.7668 \mathrm{x}+3.05$, mientras que Inga vera también expresa una tendencia de crecimiento polinómica con un $\mathrm{R}^{2}$ $=0.975 \mathrm{y}$ la ecuación que expresa su crecimiento es $\mathrm{y}=-0.0436 \mathrm{x}^{2}+0.6373 \mathrm{x}+2.492$. Las tendencias de crecimiento son similares sin embargo la mayor altura la obtuvo el tratamiento 1 Inga edulis. Tanto I. edulis como I. vera son especies cuyo principal uso es la producción de fruta. Este tipo de árboles se caracteriza por poseer un crecimiento polinomial de primer orden que se ajusta a cortos períodos de tiempo (15). Se corrobora ello en el lapso de dos años, correspondientes al ensayo, especialmente para $I$. edulis, la cual supera el crecimiento inicial de I. vera, manifestando una fase exponencial en el segundo año, donde se muestra su mayor tasa de crecimiento (Figura 1). 


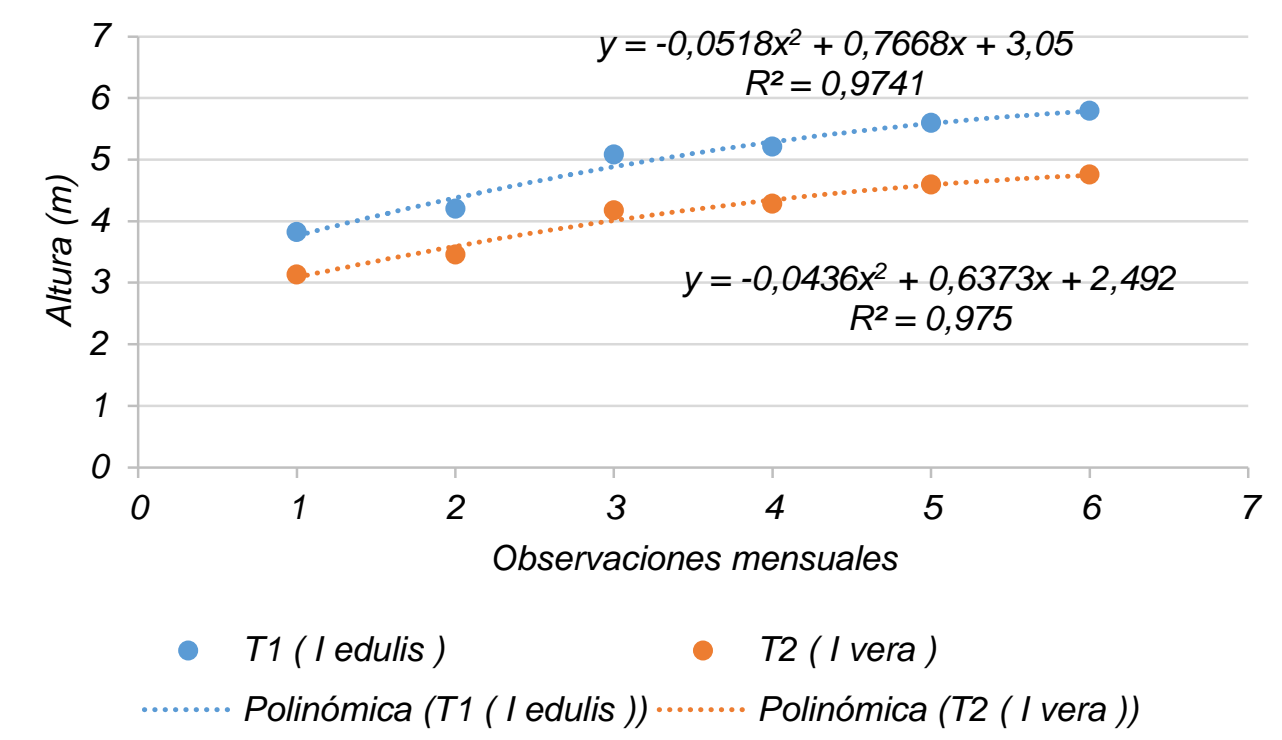

Figura 1. Análisis de regresión del crecimiento en el comportamiento fenológico de la altura de planta (m) de dos especies de Inga

Existen diferencias significativas para el diámetro altura de pecho (DAP), entre las dos especies de Inga (Tabla 3). I. edulis presenta un DAP promedio de 0.075 m superando a I. vera con un DAP promedio de $0.061 \mathrm{~m}$. No existe diferenciación de este comportamiento con ensayos sobre evaluación del crecimiento fenológico de árboles pertenecientes al género Inga, pues se espera un diámetro de 12,73 mm (0,1273 m) con una edad de diez meses (16).

Tabla 3. Comparación del diámetro altura de pecho, DAP (m), en el segundo año de establecimiento en dos especies de Inga

\begin{tabular}{lccc}
\hline \multirow{2}{*}{ Fechas } & \multirow{2}{*}{ Muestreos } & \multicolumn{2}{c}{ Medias observadas } \\
& & T1 (I edulis) & T2 (I vera) \\
\hline $15 / 07 / 19$ & 1 & 0.02 & 0.02 \\
$15 / 08 / 19$ & 2 & 0.03 & 0.02 \\
$15 / 09 / 19$ & 3 & 0.04 & 0.03 \\
$15 / 10 / 19$ & 4 & 0.05 & 0.04 \\
$15 / 11 / 19$ & 5 & 0.06 & 0.05 \\
$15 / 12 / 19$ & 6 & 0.08 & 0.06 \\
\hline
\end{tabular}


Inga edulis tiene una línea de tendencia de crecimiento polinómica con un $\mathrm{R}^{2}=0.9993$, a su vez la ecuación que expresa su crecimiento es $\mathrm{y}=0.0015 \mathrm{x}^{2}+8 \mathrm{E}-05 \mathrm{x}+0.0213$, mientras que $I$. vera también expresa una tendencia de crecimiento polinómica con un $\mathrm{R}^{2}=0.9936$ y la ecuación que expresa su crecimiento es $\mathrm{y}=0.0017 \mathrm{x}^{2}-0.0039 \mathrm{x}+0.0244$. Las tendencias de crecimiento son similares, sin embargo el mayor DAP lo obtuvo el tratamiento I. edulis (Figura 2).

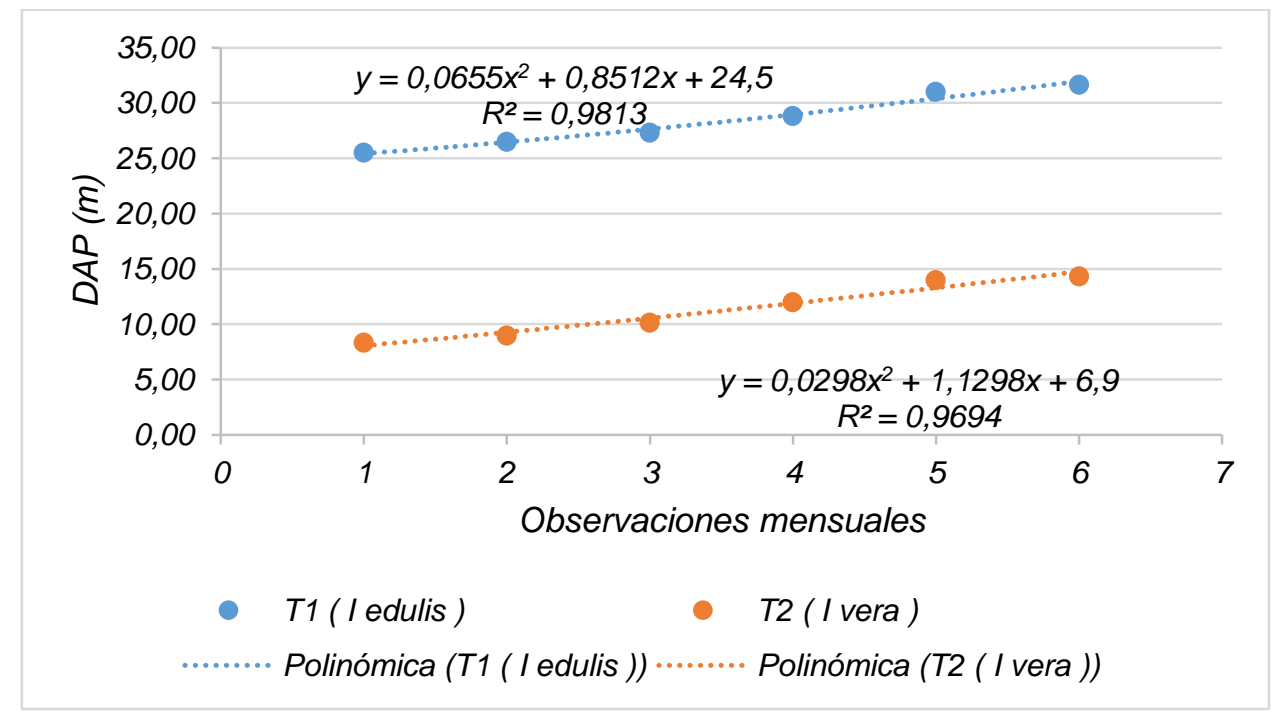

Figura 2. Análisis de regresión del crecimiento en el diámetro altura de pecho DAP (m) de dos especies de Inga

Existen diferencias significativas para la cantidad de ramas (\#), entre las dos especies de Inga. Destaca I. edulis con una cantidad de ramas promedio de 31.66, superando a I. vera que alcanza 14.33 ramas promedio. Con respecto a la producción de ramas, existen estudios (14) que sostienen que $I$. edulis tiene una generación continua y fisiológicamente más activa que otras especies del género, produciendo mayor ramaje en épocas de picos lluviosos. Este fenómeno fue apreciado en el comportamiento de las especies evaluadas (Tabla 4).

Tabla 4. Comparación de la cantidad de ramas (\#), en el segundo año de establecimiento en dos especies de Inga

\begin{tabular}{lccc}
\hline \multirow{2}{*}{ Fechas } & \multirow{2}{*}{ Muestreos } & \multicolumn{2}{c}{ Medias observadas } \\
& & T1 (I edulis) & T2 (I vera) \\
\hline $15 / 07 / 19$ & 1 & 25.50 & 8.33 \\
$15 / 08 / 19$ & 2 & 26.50 & 9.00 \\
$15 / 09 / 19$ & 3 & 27.33 & 10.17 \\
$15 / 10 / 19$ & 4 & 28.83 & 12.00 \\
$15 / 11 / 19$ & 5 & 31.00 & 14.00 \\
$15 / 12 / 19$ & 6 & 31.67 & 14.33 \\
\hline
\end{tabular}


La especie Inga edulis tiene una línea de tendencia de crecimiento polinómica con un $\mathrm{R}^{2}=$ 0.9813 , a su vez la ecuación que expresa su crecimiento es $\mathrm{y}=\mathrm{y}=0.0655 \mathrm{x}^{2}+0.8512 \mathrm{x}+24.5$, mientras que $I$. vera también expresa una tendencia de crecimiento polinómica con un $\mathrm{R}^{2}=0.9694 \mathrm{y}$ la ecuación que expresa su crecimiento es $\mathrm{y}=0.0298 \mathrm{x}^{2}+1.1298 \mathrm{x}+6.9$. Las tendencias de crecimiento son similares sin embargo la mayor cantidad de ramas la obtuvo I. edulis (Figura 3).

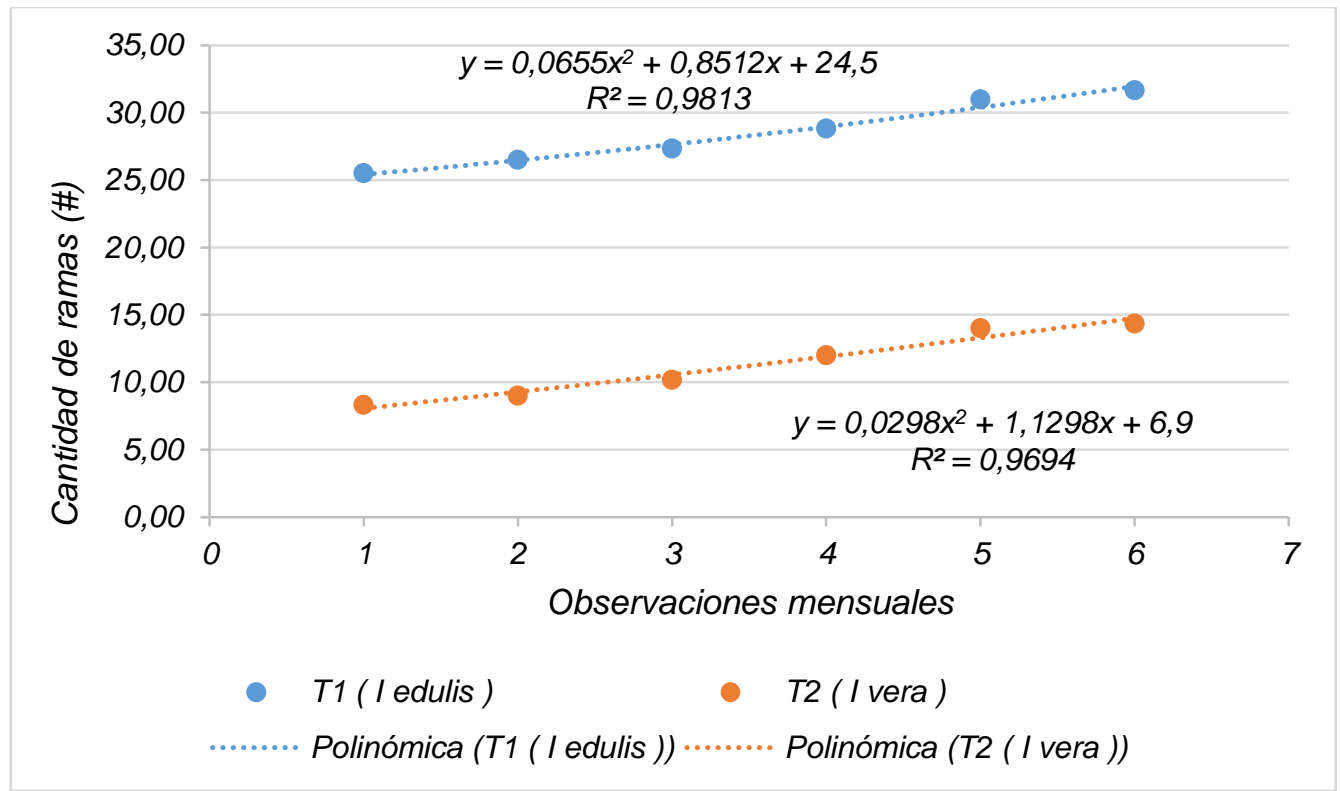

Figura 3. Análisis de regresión de la cantidad de ramas (\#) de dos especies de Inga

Se puede observar que existen diferencias significativas para el diámetro de área con sombreamiento $(\mathrm{m})$, entre las dos especies de Inga, superando edulis, con un diámetro promedio de $5.94 \mathrm{~m}$ a $I$. vera, la misma que llega a un promedio de $4.79 \mathrm{~m}$ (Tabla 5).

Tabla 5. Comparación del diámetro de área con sombreamiento (m), en el segundo año de establecimiento en dos especies de Inga.

\begin{tabular}{lccc}
\hline \multirow{2}{*}{ Fechas } & \multirow{2}{*}{ Muestreos } & \multicolumn{2}{c}{ Medias observadas } \\
& & T1 (I edulis) & T2 (I vera) \\
\hline $15 / 07 / 19$ & 1 & 3.07 & 2.75 \\
$15 / 08 / 19$ & 2 & 3.54 & 3.03 \\
$15 / 09 / 19$ & 3 & 5.12 & 4.37 \\
$15 / 10 / 19$ & 4 & 5.63 & 4.80 \\
$15 / 11 / 19$ & 5 & 5.68 & 4.85 \\
$15 / 12 / 19$ & 6 & 5.78 & 4.88 \\
\hline
\end{tabular}


En la Figura 4 se puede observar que Inga edulis tiene una línea de tendencia de crecimiento polinómica con un $\mathrm{R}^{2}=0.9505$, a su vez la ecuación que expresa su crecimiento es $\mathrm{y}=\mathrm{y}=-0.1418 \mathrm{x}^{2}+1.5778 \mathrm{x}+1.4305$, mientras que $I$. vera también expresa una tendencia de crecimiento polinómica con un $\mathrm{R}^{2}=0.9349$ y la ecuación que expresa su crecimiento es $\mathrm{y}=-0.1148 \mathrm{x}^{2}+1.2767 \mathrm{x}+$ 1.3848. Las tendencias de crecimiento son similares, sin embargo el mayor sombreamiento lo obtuvo I. edulis. Este resultado concuerda con los parámetros esperados, ya que, basándose en el desarrollo observado de sus ramas laterales, I. edulis puede considerarse una planta más promisoria para reforestación o como generadora de sombra para otros cultivos (17).

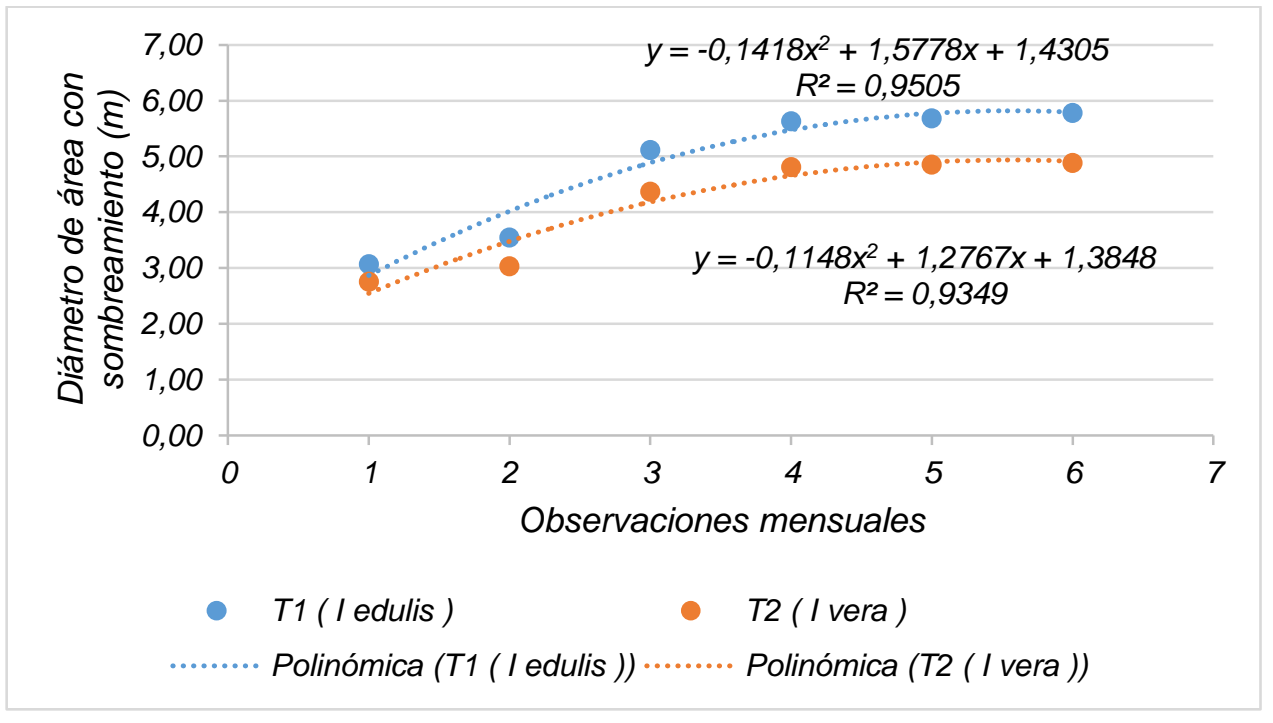

Figura 4. Análisis de regresión del diámetro de área con sombreamiento (m) de dos especies de Inga

Cuantificación de los nutrientes en las hojas y aporte edáfico de nitrógeno en el área de influencia de Inga edulis $e$ I. vera, en la zona El Vainillo, El Triunfo,

\section{Guayas, Ecuador}

En la Tabla 6 se puede observar que existen diferencias significativas para el peso en gramos de las hojas expresado en materia seca ajustado a menos $10 \%$ de humedad. Inga edulis llega a tener una media de peso de hojarasca por árbol de 380.78 g, cuyo valor supera a $I$. vera que alcanza 100.92 g. Trabajos realizados han demostrado mayor cantidad y peso de hojas en I. edulis (14). Sin embargo, la vida de las hoja de I. edulis es más corta que I. vera (18), por lo que ambas especies, aunque experimenten caída de hojas, las de I. edulis se descomponen con más rapidez; estudios afirman que esta característica se presenta a los dieciocho meses de edad (14), pero este fenómeno pudo apreciarse desde las primeras etapas de desarrollo de ambas especies. Las hojas de $I$. vera tienen mayores dimensiones, aunque su peso una vez secas y caídas en el suelo es menor que las hojas de I. edulis (19). 
Tabla 6. Comparación del peso (g) de las hojas/árbol expresado en materia seca ajustado a menos 10\%, en el segundo año de establecimiento en dos especies de Inga

\begin{tabular}{lccc}
\hline \multirow{2}{*}{ Fechas } & \multirow{2}{*}{ Muestreos } & \multicolumn{2}{c}{ Medias observadas } \\
& & T1 (I edulis) & T2 (I vera) \\
\hline $15 / 07 / 19$ & 1 & 125.02 & 32.67 \\
$15 / 08 / 19$ & 2 & 147.27 & 44.63 \\
$15 / 09 / 19$ & 3 & 225.86 & 68.19 \\
$15 / 10 / 19$ & 4 & 277.83 & 84.58 \\
$15 / 11 / 19$ & 5 & 305.56 & 93.02 \\
$15 / 12 / 19$ & 6 & 380.78 & 100.92 \\
\hline
\end{tabular}

La especie Inga edulis tiene una línea de tendencia de crecimiento polinómica con un $\mathrm{R}^{2}=$ 0.9818, a su vez la ecuación que expresa su crecimiento es $y=1.0968 x^{2}+43.913 x+73.387$, mientras que Inga vera también expresa una tendencia de crecimiento polinómica con un $\mathrm{R}^{2}=$ 0.9875 y la ecuación que expresa su crecimiento es $\mathrm{y}=-1.4425 \mathrm{x}^{2}+24.465 \mathrm{x}+6.9197$. Las tendencias de crecimiento son similares sin embargo el mayor peso de las hojas expresado en materia seca ajustado a menos 10\% para condiciones reales lo obtuvo Inga edulis (Figura 5).

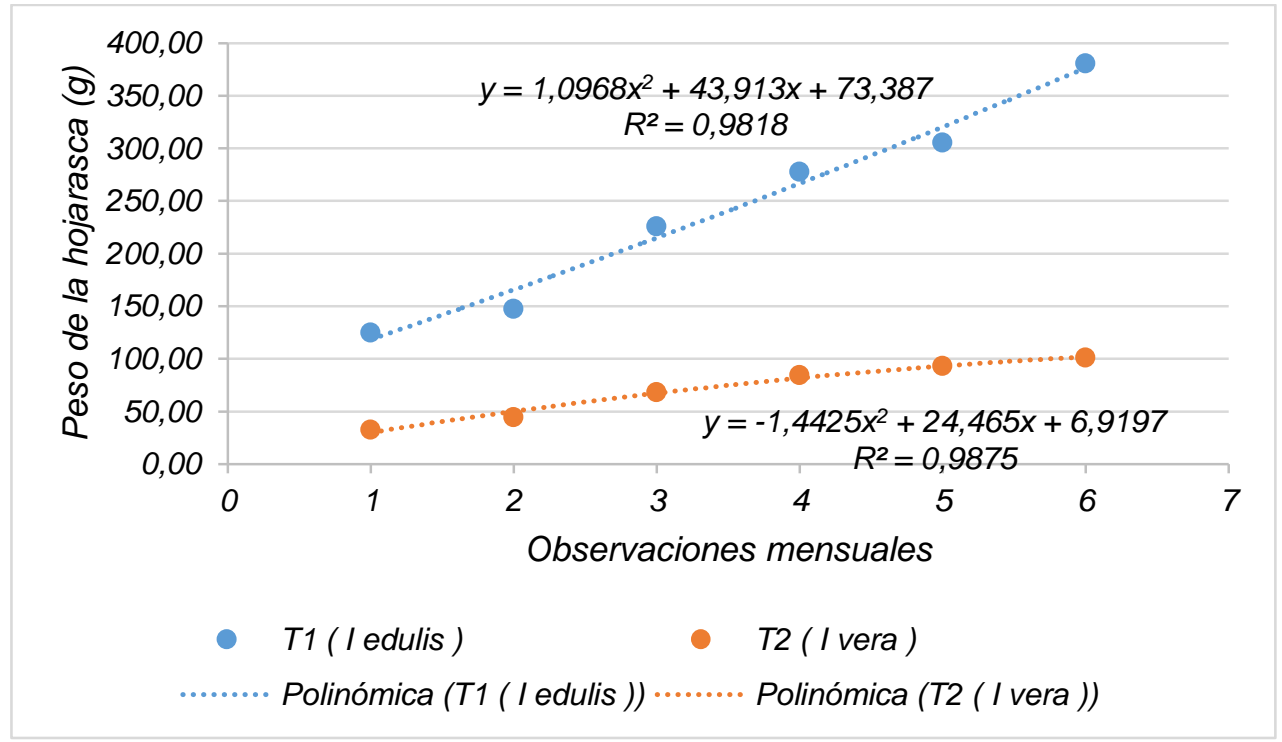

Figura 5. Análisis de regresión de la producción de hojarasca expresado en materia seca (g) ajustado a menos $10 \%$ para condiciones reales de dos especies de Inga

Luego de haber efectuado un análisis foliar de la hojarasca, como promedio Inga edulis posee $3.13 \%$ de $\mathrm{N}$, mientras que $I$. vera tiene $2,53 \%$ de N. Sin embargo es de resaltar que la especie Inga vera a pesar de poseer un número inferior en cuanto a nitrógeno supera a la especie I. edulis en elementos como potasio y calcio (Tabla 7). 
Tabla 7. Cuantificación el porcentaje de nitrógeno en las hojas de dos especies de Inga

\begin{tabular}{lccccc}
\hline \multirow{2}{*}{ Especie } & \multicolumn{5}{c}{ Concentración foliar (\%) } \\
\\
\hline T1 (I edulis) & $\mathbf{N}$ & $\mathbf{K}$ & $\mathbf{P}$ & $\mathbf{C a}$ & $\mathbf{M g}$ \\
T2 (I vera) & 2,13 & 0,63 & 0,11 & 0,43 & 0,31 \\
\hline
\end{tabular}

Todas las especies conocidas de Inga producen nódulos en las raíces los cuales contienen bacterias fijadoras de nitrógeno y las raíces Inga se asocian con hongos micorrizas (12). Entre mayor sea la biomasa de la especie de Inga, mayor va a ser la cantidad de nódulos que desarrolla cada árbol (20).

Tabla 8. Aporte edáfico de nitrógeno en el área de influencia de los árboles de dos especies de Inga

\begin{tabular}{lccccccc}
\hline Fechas & Muestreo & \multicolumn{2}{c}{$\begin{array}{c}\text { Medias observadas } \\
\text { peso hoja seca (g) } \\
\text { ajustados }\end{array}$} & $\begin{array}{c}\text { Medias calculadas } \\
\text { peso hoja seca (Kg) } \\
\text { por hectárea }\end{array}$ & \multicolumn{2}{c}{$\begin{array}{c}\text { Medias calculadas } \\
\text { peso nitrógeno (Kg) } \\
\text { por ha }\end{array}$} \\
& & $\begin{array}{c}\text { T1 } \\
\text { (I. edulis) }\end{array}$ & $\begin{array}{c}\text { T2 } \\
\text { (I. vera) }\end{array}$ & $\begin{array}{c}\text { T1 } \\
\text { (I. edulis) }\end{array}$ & $\begin{array}{c}\text { T2 } \\
\text { (I. vera) }\end{array}$ & $\begin{array}{c}\text { T1 } \\
\text { (I. edulis) }\end{array}$ & $\begin{array}{c}\text { T2 } \\
\text { (I. vera) }\end{array}$ \\
\hline $15 / 07 / 2019$ & 1 & 125.02 & 32.67 & 34.76 & 9.08 & 1.09 & 0.88 \\
$15 / 08 / 2019$ & 2 & 147.27 & 44.63 & 40.94 & 12.41 & 1.28 & 1.04 \\
$15 / 09 / 2019$ & 3 & 225.86 & 68.19 & 62.79 & 18.96 & 1.97 & 1.59 \\
$15 / 10 / 2019$ & 4 & 277.83 & 84.58 & 77.24 & 23.51 & 2.42 & 1.95 \\
$15 / 11 / 2019$ & 5 & 305.56 & 93.02 & 84.95 & 25.86 & 2.66 & 2.15 \\
$15 / 12 / 2019$ & 6 & 380.78 & 100.92 & 105.86 & 28.06 & 3.31 & 2.68 \\
Valor 12 meses & & 4569.36 & 1211.04 & 1270.28 & 336.67 & 39.76 & 32.14 \\
\hline
\end{tabular}

Asumiendo que el tratamiento 1, durante 12 meses, obtendría una sumatoria de $4569.36 \mathrm{~g}$ de materia seca ajustada al menos $10 \%$ por cada árbol, y que una hectárea posee 278 árboles, se tendría $1270.28 \mathrm{~kg}$ de hoja seca/ha/año, la misma que contiene $3.13 \%$ de $\mathrm{N}$; por lo tanto, en relación a la cantidad de materia seca de hojarasca se tendría 39.76 kg de N/ha/año. Mientras que el tratamiento 2 durante 12 meses obtendría $1211.04 \mathrm{~g}$ de materia seca, que corresponde, con los mismos parámetros, a $336.67 \mathrm{~kg}$ de hoja seca/ha/año, la misma que corresponde a $32.14 \mathrm{~kg}$ de $\mathrm{n} / \mathrm{ha} / a$ ño. Ello indica que el mayor aporte edáfico al suelo por hojarasca seca se obtiene con I. edulis. 
Las plantas leguminosas reducen el $\mathrm{N}$ atmosférico a formas asimilables para las plantas, como el amonio $\left(\mathrm{NH}_{4}\right)$, siendo este el principal nutriente (21). Todo proceso de fijación de nitrógeno y su posterior retorno al suelo, debe evidenciarse en los contenidos de nitrógeno foliares (22). Cualquier variación en este aspecto se debe a la mayor producción de biomasa foliar, pero también a la concentración de elementos en los tejidos de las hojas (23).

\section{Fauna benéfica en el área de influencia de Inga edulis e I. vera, en la zona El Vainillo, El Triunfo, Guayas, Ecuador}

A los 24 meses de establecimiento de los árboles, se realizó la identificación de organismos benéficos en el área de influencia Inga edulis e I. vera. A nivel de suelo, a una profundidad de 0,30 $\mathrm{m}$ en un área de $1 \mathrm{~m} 2 \mathrm{se}$ contabilizaron los individuos de Lumbricus terrestres (orden Crassiclitellata, familia Lumbricidae), mientras que en el dosel de ramas se realizó observación, sin captura, de la cantidad de Polistes dominula y Vespula vulgaris (orden Hymenoptera, familia Vespidae) habitando los árboles muestreados (Figura 6). La presencia de fauna benéfica se deriva del hábitat conformado por los cultivos de sombra, que sirve como refugio de la fauna silvestre (7).

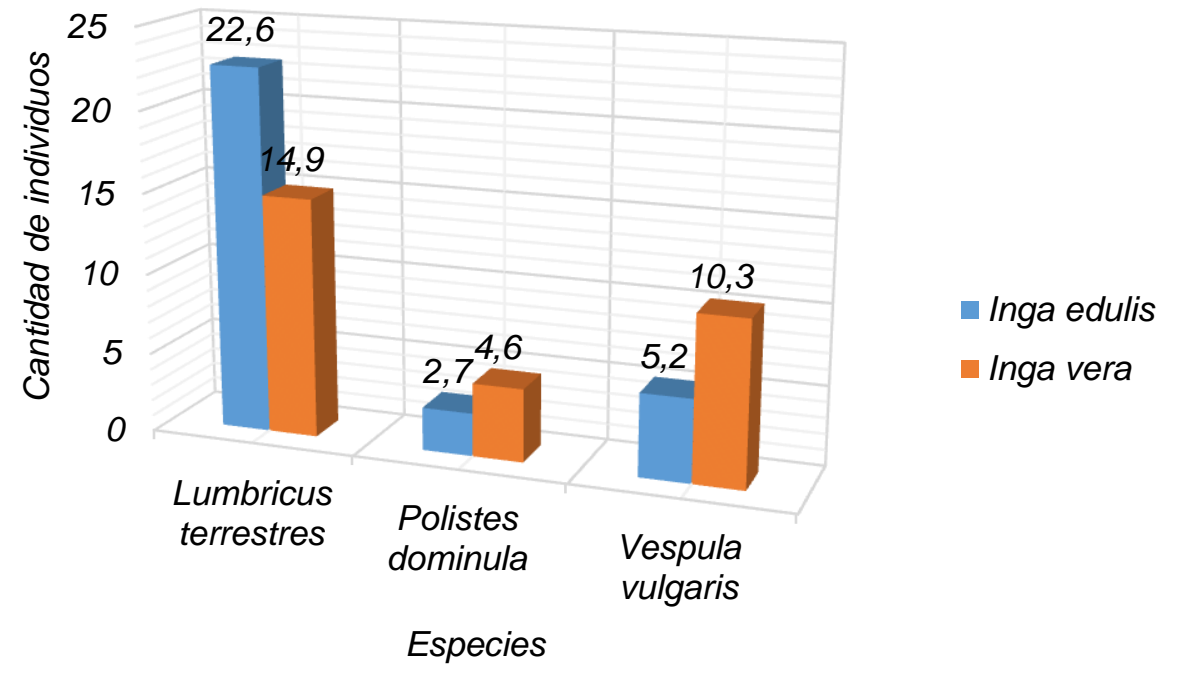

Figura 6. Fauna benéfica en el área de influencia de los árboles de dos especies de Inga

La población de Lumbricus terrestres fue mayor en I. edulis (22,6 individuos promedio) que en $I$. vera $(14,9$ individuos). Las relaciones de las lombrices de tierra con los sistemas radiculares de plantas han sido reportados en estudios (24), evidenciando que raíces de mayor tamaño crean un mejor hábitat para las lombrices, lo que en efecto pudo ser corroborado pues el sistema radicular de I. edulis es más desarrollado que el de I. vera. Destacó el hecho que, pese a su menor ramaje, en $I$. vera se detectaron más individuos de $P$. dominula y $V$. vulgaris $(4,6$ y 10,3 en promedio), con respecto a I. edulis, 
donde tan solo se detectaron un promedio de 2,7 y 5,2 ( $P$. dominula y $V$. vulgaris, respectivamente). El comportamiento fenológico de las especies sembradas apoya los resultados del muestreo, pues las avispas, al ser entes mayormente solitarios $y$ territoriales (25), aprovechan mejor las dimensiones grandes de las hojas de I. vera y el menor tamaño del árbol, que les confiere un ambiente exclusivo y propicio para su establecimiento, sintiéndose protegidas, $\mathrm{y}$ colonizando en menor medida a I. edulis cuyas hojas son más pequeñas y con mayor separación de las ramas.

\section{CONCLUSIONES}

De acuerdo a los resultados obtenidos en este estudio, se llegó a las siguientes conclusiones:

Se demostró que, hasta los dos años de establecimiento, la línea de crecimiento de las especies I. edulis e I. vera es polinómica en altura, diámetro, ramaje y sombreamiento $\left(\mathrm{R}^{2}=\right.$ 0.9). I. edulis superó a I. vera en todos indicadores de desarrollo fenológico analizados. Además es mayor su aporte edáfico de $\mathrm{NH}_{4}$, alcanzando $39.76 \mathrm{~kg} \mathrm{~N} / \mathrm{ha}$ /año contra los $32.14 \mathrm{~kg}$ N/ha/año a los que llega I. vera.

Realizado un análisis foliar de macronutrientes, se evidencia un comportamiento diferente entre estas dos especies. El porcentaje en elementos como $\mathrm{P}$ $(11 \%)$ y $\mathrm{Mg}(0,31 \%)$ es mayor a nivel foliar en $I$. edulis, con respecto a I. vera $(9 \%$ y $16 \%$, respectivamente), teniendo esta última mejores concentraciones foliares de $\mathrm{K}(0,79 \%)$ y Ca $(1 \%)$ que superan a I. edulis $(63 \% \mathrm{~K}$ y $0,43 \% \mathrm{Ca}$ ).

La presencia de lombrices de tierra (Lumbricus terrestres) en el área del subsuelo con influencia de la raíz, difiere en estas especies. Se detectó una población promedio de 22,6 individuos de L. terrestres en I. edulis, que es superior a los encontrados en I. vera $(14,9)$.

A nivel de fuste es característica la presencia de avispas, siendo identificadas dos especies: Polistes dominula y Vespula vulgaris. En este caso, también se exhiben diferencias, pues la población promedio de individuos de $P$. dominula $(4,6)$ y $V$. vulgaris $(10,3)$ en $I$. vera es mayor que la detectada en $I$. edulis, donde, respectivamente, se detectaron 2,7 y 5,2 individuos.

\section{REFERENCIAS}

1. Penold, C. y Collins, C. Cover crops and weed suppression. Adelaida, Australia: The University of Adelaide. Fac Tsheet. (2012). Consultado 20 diciembre de 2019. Disponible en: http://www.mvwi.com.au/items/473/2 012-06-FS-Cover-Crops-Weed-

Suppression.pdf

2. FAO. Perspectivas a plazo medio de los productos básicos agrícolas. ISBN 92-5305077-2. Roma, Italia: Organización de las Naciones Unidas para la Agricultura y la Alimentación (FAO). (2014). Consultado 20 diciembre de 2019. Disponible en: http://www.fao.org/docrep/007/y5143 s/y5143s0w.htm

3. Toomsaw, B., Cadisch, M., Srichantawong, M., Thongsodsaeng, C., Giller, K. y Limpinuntann, V. Biological N2 fixation and residual $\mathrm{N}$ benefit of pre-rice leguminous crops and green manures. Netherlands Journal of Agricultural Science, (2010). 48(1), 19-29.

4. Schoeneberger, M. Trabajos de agroforestería para el secuestro de carbono en las tierras agrícolas. Sistemas Agroforestales. Madrid, España: Rainforest Saver. (2009).

5. Checa, X. y Grijalva, J. Situación de los recursos genéticos forestales en Ecuador. Quito, Ecuador: Instituto Nacional de Investigaciones Agropecuarias (INIAP), (2012). 
6. Leng, R. Sistemas Intensivos para la Producción Animal y de Energía Renovable con Recursos Tropicales. Cali, Colombia: Centro para la Investigación en Sistemas Sostenibles de Producción Agropecuaria (CIPAV), (2008).

7. Botero, R. y Russo, R. Utilización de árboles y arbustos fijadores de nitrógeno en sistemas sostenibles de producción animal en suelos ácidos tropicales. San José de Costa Rica: Agroforestería para la Producción Animal en Latinoamérica. (2000).

8. INIBAP. Producción orgánica y/o ambientalmente amigable. Guácimo, Costa Rica: EARTH. (2000).

9. Pennington, T. y Fernández, E. El género Inga: Utilización. Bruselas, Bélgica: Continental Printing.(2002).

10. De Los Ángeles, A. Evaluación de crecimiento inicial en tres especies del género Inga en sistema agroforestal. Lima, Perú: Universidad Politécnica de Valencia. (2013).

11. Hands, M. Usos de Inga en suelos ácidos de zonas selváticas: sostenibilidad del cultivo en franjas y regeneración del suelo. San Salvador, El Salvador: Fundesyra. (2000).

12. Valle, G. Manual agroforestal del Inga (Inga edulis). Tegucigalpa, Honduras: Centro Universitario Regional del Litoral Atlántico (CURLA) (2010).

13. FAO. Especies forestales productoras de frutas $y$ otros alimentos. Roma, Italia: Food and Agriculture Organization of the United Nations (FAO). (2016).

14. Marín, O., Castaño, A. y Gómez, G. Fenología del guamo Inga edulis (Fabales: Mimosoideae) en dos agroecosistemas del Quindío, Colombia. Revista de Investigaciones de la Universidad del Quindío (RIUQ), (2012). 23(2), 127-133. http://dx.doi.org/10.15517/ma.v29i2.28 759

15. Escobar, C., Zuluzaga, J. y Martínez, A. El cultivo de arazá (Eugenia stipitata). Caquetá, Colombia: Corporación Colombiana de Investigación Agropecuaria (CORPOICA). (1996).
16. Abril, R., Ruiz, T., Alonso, J., Cabrera, G. y Meric, O. Crecimiento inicial de Eugenia stipitata, Inga spectabilis e Inga edulis en Napo, Ecuador. Agronomía Mesoamericana, (2018). 29(2), 275-291. http://dx.doi.org/10.15517/ma.v29i2.28 759

17. Falcao, M. y Clement, C. (2000). Fenologia e produtividade do Infá-Cipó (Inga edulis) na Amazônia Central. Acta Amazónica, $\quad 30(1), \quad$ 173-180. http://doi:10.1590/180943922000302180

18. Monro, A., Velarde, R., Flores, R., Soruco, V., Reyes, J. y Miliken, W. Manual agroforestería Inga. Chicago, USA: Royal Botanic Gardens, Kew \& Herencia, (2016).

19. Bressani, R. Valoración química nutricional de la harina de semilla de diferentes especies de Inga (I. jinucuil, I. laurina, I. vera). Estudios preliminares para su incorporación en la dieta de la población rural. Guatemala: Universidad del Valle de Guatemala. Consejo Nacional de Ciencia y Tecnología (CONCYT). Secretaría Nacional de Ciencia y Tecnología (SENACYT). Fondo Nacional de Ciencia y Tecnología (FONACYT). Proyecto FODECYT No. 043-2006, (2010).

20. Puertas, F., Arévalo, E., Zúñiga, L., Alegre, J., Loli, O., Soplin, H. y Baligar, VEstablecimiento de cultivos de cobertura y extracción total de nutrientes en un suelo de trópico húmedo en la Amazonía Peruana. Ecología Aplicada, (2008). 7(12), 23-28

21. Ovalle, C., Del Pozo, A., Lavin, A. y Hirzel, J. Cover crops in vineyards: performance of annual forge legume mixtures and effects on soil fertility. Chilean Journal of Agricultural Research, (2007). 67(1), 384-392

22. Flower, K., Cordingley, N., Warda, P. y Weeks, C. Nitrogen, weed management and economics with cover crops in conservation agriculture in a Mediterranean climate. Field Crops Research, (2012). 132(1), 63-75 
23. Marinho, G., Ndiae, A., Linhares, R. y Azevedo, J. Cultivos de cobertura como indicadores de procesos ecológicos. LEISA. Revista de Agroecología, (2007). 22(4), 20-22

24. Ydrogo, H., Ruíz, P., Pashanasi, B. y Lavalle, P. Inoculación de lombrices de tierra Pontoscolex corethrurus y presencia de micorrizas Vesículo arbusculares en plántulas de arazá (Eugenia stipitata), achiote (Bixa orellana), y pijuayo (Bactris gasipaes) y sus efectos en el crecimiento. Folia Amazónica, (1995). 7(1-2), 5-14

25. Vanoye, M., Meléndez, V., Ayala, R., Navarro, J. y Delfín, H. Avispas depredadoras de áreas naturales protegidas del estado de Yucatán, México. Revista Mexicana de Biodiversidad, (2015). 86(4), 989-997. https://dx.doi.org/10.1016/j.rmb.2015.0 4.037 